\title{
Wild edible mushrooms, a valuable resource for food security and rural development in Burundi and Rwanda
}

\author{
Jérôme Degreef ${ }^{(1,2)}$, Laurent Demuynck ${ }^{(3)}$, Assumpta Mukandera ${ }^{(4)}$, \\ Gudula Nyirandayambaje ${ }^{(5)}$, Benoît Nzigidahera ${ }^{(6)}$, André De Kesel ${ }^{(1)}$ \\ (1) Botanic Garden Meise. Nieuwelaan, 38. BE-1860 Meise (Belgium).E-mail: jerome.degreef@botanicgardenmeise.be \\ (2) Fédération Wallonie-Bruxelles. Service Général de l'Enseignement universitaire et de la Recherche scientifique. Rue \\ Lavallée, 1. BE-1080 Bruxelles (Belgium). \\ ${ }^{(3)}$ Kigali Farms - Mundi Center. Rwandex. Kicukiro. PO Box 1796. Kigali (Rwanda). \\ (4) University of Rwanda. College of Education. Remera Campus. KG 11. Avenue 47. PO Box 5039. Kigali (Rwanda). \\ ${ }^{(5)}$ Karisoke Research Center. Dian Fossey Gorilla Fund International. PO Box 105. Ruhengeri-Musanze (Rwanda). \\ (6) Office Burundais pour la Protection de l'Environnement. Quartier Musinzira. BP 56. Gitega (Burundi).
}

Received on October 16, 2015; accepted on May 26, 2016.

Description of the subject. This study is the first to compile the diversity of wild edible mushrooms gathered in the different ecosystems (savanna, woodland, montane forest, and exotic tree plantations) of Burundi and Rwanda and to consider this resource in a developmental framework for the region.

Objectives. The aim of this study is to update the inventory of wild edible mushrooms in Burundi and Rwanda focusing on their sustainable exploitation and their potential for cultivation.

Method. The inventory is based on a literature survey completed with referenced specimens gathered during field trips between 2010 and 2015 that are deposited at the Botanic Garden Meise (BR).

Results. Seventy-seven species of edible mushrooms have been listed together with their habitat and ecology. This includes 39 new records of species eaten in the region, 8 of which were not previously known to be edible. An attempt to combine the protection of natural ecosystems with the development of local populations is presented in relation to the availability of these mushrooms, their ecology and cultivation potential.

Conclusions. Wild edible mushrooms constitute an interesting and under-exploited resource in Burundi and Rwanda. Sustainable gathering of ectomycorrhizal species in miombo woodlands and cultivation of saprotrophic species from montane forests suggest important food issues and interesting business perspectives.

Keywords. Non-wood forest products, fungi, human nutrition, storage, tropical Africa.

Les champignons sauvages comestibles, une ressource précieuse pour la sécurité alimentaire et le développement rural au Burundi et au Rwanda

Description du sujet. Cette étude est la première à présenter la diversité des champignons sauvages comestibles collectés dans les différents écosystèmes (savane, forêt claire, forêt de montagne et plantation d'arbres exotiques) du Burundi et du Rwanda et à considérer cette ressource dans le cadre du développement de la région.

Objectifs. L'objectif de cette étude est de mettre à jour l'inventaire des champignons sauvages comestibles du Burundi et du Rwanda en mettant l'accent sur leur exploitation durable et sur leur potentiel de mise en culture.

Méthode. L'inventaire se base sur une étude bibliographique complétée par des échantillons de référence collectés lors de missions de terrain menées de 2010 à 2015 et conservés à l'herbier du Jardin botanique de Meise (BR).

Résultats. Un inventaire de 77 espèces de champignons comestibles a été établi, complété d'informations sur leurs habitats et leur écologie. Parmi celles-ci, 39 sont pour la première fois signalées comme consommées dans la région et 8 n'étaient pas connues comme comestibles jusqu'à présent. Des considérations visant à concilier la protection des écosystèmes naturels et le développement des populations locales sont présentées en relation avec la disponibilité de ces champignons, leur écologie et leur aptitude à être cultivés.

Conclusions. Les champignons sauvages constituent une ressource intéressante et sous-exploitée au Burundi et au Rwanda. La mise en place de collectes raisonnées pour les espèces ectomycorhiziennes des forêts claires de type miombo et d'unités de production pour les espèces saprotrophes des forêts de montagne laisse entrevoir d'importants enjeux en termes de nutrition et d'intéressantes perspectives économiques.

Mots-clés. Produits forestiers non ligneux, champignon, nutrition humaine, stockage, Afrique tropicale. 


\section{INTRODUCTION}

Malnutrition is a major concern in Burundi and Rwanda. Household food security still constitutes a challenge in the region despite some recorded improvement by the UNDP (Human Development Report 2014, www.wfp. org, accessed 8 Sept 2015). Burundi and Rwanda have the highest population densities in Africa and face significant food deficits. In particular, the Burundian provinces of the North and East, and the northern and western areas of Rwanda bordering Lake Kivu and along the Congo Nile Crest, are the most affected. This food deficit cannot be met by the local agriculture that is mainly in the hands of small subsistence farmers. It is precisely these poor rural households dependent on low income agriculture or casual labor that face food insecurity.

In Africa, over two thirds of the population rely on forest products, either in the form of subsistence or as a cash income derived from a wide range of Non-Timber Forest Products (NTFPs), including edible mushrooms (Rammeloo et al., 1993). The diversity of the latter has been widely documented for West- (Yorou et al., 2001a; Yorou et al., 2001b; De Kesel et al., 2002; Ducousso et al., 2003; Guissou et al., 2008; Yorou et al., 2014), central- (Mossebo et al., 2002; van Dijk et al., 2003; Malaisse et al., 2008; Eyi Ndong et al., 2010; Eyi Ndong et al., 2011) and East-Africa (Pegler et al., 1980; Piearce, 1981; Chipompha, 1985; Morris, 1987; Morris, 1990; Härkönen et al., 1993; Härkönen et al., 1994; Härkönen et al., 1995; Degreef et al., 1997; Malaisse, 1997; Karhula et al., 1998; Härkönen et al., 2003; De Kesel et al., 2010; Härkönen et al., 2015).

The only available records of edible mushrooms for the studied region are the preliminary inventories of the remaining Burundian miombo woodlands near Lake Tanganyika and along the Tanzanian border (Buyck, 1994; Buyck et al., 1995; Nzigidahera, 2007). Until now, no data have been gathered in the montane forests of the Congo-Nile Divide region of Burundi and Rwanda. Owing to the scarcity of natural ecosystems and the restricted access to National Parks, mushrooming and consumption of wild species have progressively and inevitably declined in both countries. However, a number of these species are probably good candidates for cultivation and food biomass production.

It is well known that mushroom cultivation is a cost-effective way to provide proteins i.e. requiring minimal land, temperate climate, high humidity and waste organic substrates. Despite most of these conditions being met, several attempts to grow exotic mushrooms in Burundi and Rwanda have failed. We think that a low-tech and low-cost approach to the cultivation of endemic/local edible fungi may be more successful. This paper presents a full list of the edible species known from the region, including the ones with a high potential for successful cultivation.

\section{MATERIALS AND METHODS}

\subsection{Study area}

Mushroom collecting (2010-2015) was conducted in various ecosystems in Burundi (Jan. 2011, Réserve Naturelle Forestière Vyanda/Rumonge, S $03.9958^{\circ}$ $04.0273^{\circ} /$ E $29.4720^{\circ}-29.5187^{\circ}$, miombo woodland; Nov. 2013, Parc National de la Kibira, S $02.8032^{\circ}$ $03.2816^{\circ} /$ E $29.3716^{\circ}-29.5915^{\circ}$, montane forest; Paysage protégé de Gisagara, S $03.2161^{\circ}-03.2586^{\circ}$ / E $30.7342^{\circ}-30.7679^{\circ}$, miombo woodland), and Rwanda (Dec. 2010, Rulindo district, S $01.7525^{\circ}-01.8511^{\circ} /$ E $29.9600^{\circ}-30.0036^{\circ}$, savanna; Dec. 2010, Nyungwe National Park, S $02.2510^{\circ}-03.2811^{\circ} /$ E $29.0495^{\circ}$ $29.5874^{\circ}$, montaneforest; March2011,Bugeseradistrict, S $02.1188^{\circ}-02.2308^{\circ} /$ E $30.1919^{\circ}-30.4237^{\circ}$, savanna; Oct. 2014, Nyungwe National Park, S 02.2510$03.2811^{\circ} / \mathrm{E} 29.0495^{\circ}-29.5874^{\circ}$, montane forest; April 2015, Gishwati National Park, S 01.6247º-01.9010 / E 29.3538 $-29.5038^{\circ}$ and Volcanoes National Park, S $01.3549^{\circ}-01.5568^{\circ} /$ E $29.3677^{\circ}-29.7045^{\circ}$, montane forest). The sampling areas covered most of the remaining miombo woodlands and montane forests found in Burundi and Rwanda (Figure 1).

\subsection{Data collection and analysis}

National Park rangers, local guides and villagers from the Batwa community who are familiar with collecting edible mushrooms accompanied every field trip to record local knowledge (e.g. palatability). The reliability of our findings on edibility was ensured by comparing the information obtained from Batwa guides accompanying the collecting trips in Burundi and Rwanda between 2010 and 2015. Sampling in the region (localities mentioned above) focused not only on mushrooms eaten locally but also on species consumed in other tropical African countries cited in literature.

Field methodology for collecting, photographing, describing, preparing and storing reference herbarium specimens followed best practice described by Buyck et al. (2010) and Eyi Ndong et al. (2011). Basidiomes collected were photographed in situ. Fresh impermanent features (colour, taste, scent) and morphological characters were carefully documented in the field. Specimens were cut into slices and dried using a field dryer at a temperature of $\sim 50{ }^{\circ} \mathrm{C}$ (De Kesel, 2001). Microscopic examinations were carried out using an Olympus BX51 microscope from rehydrated herbarium material mounted in $3 \% \mathrm{KOH}$ and Congo Red solutions. 


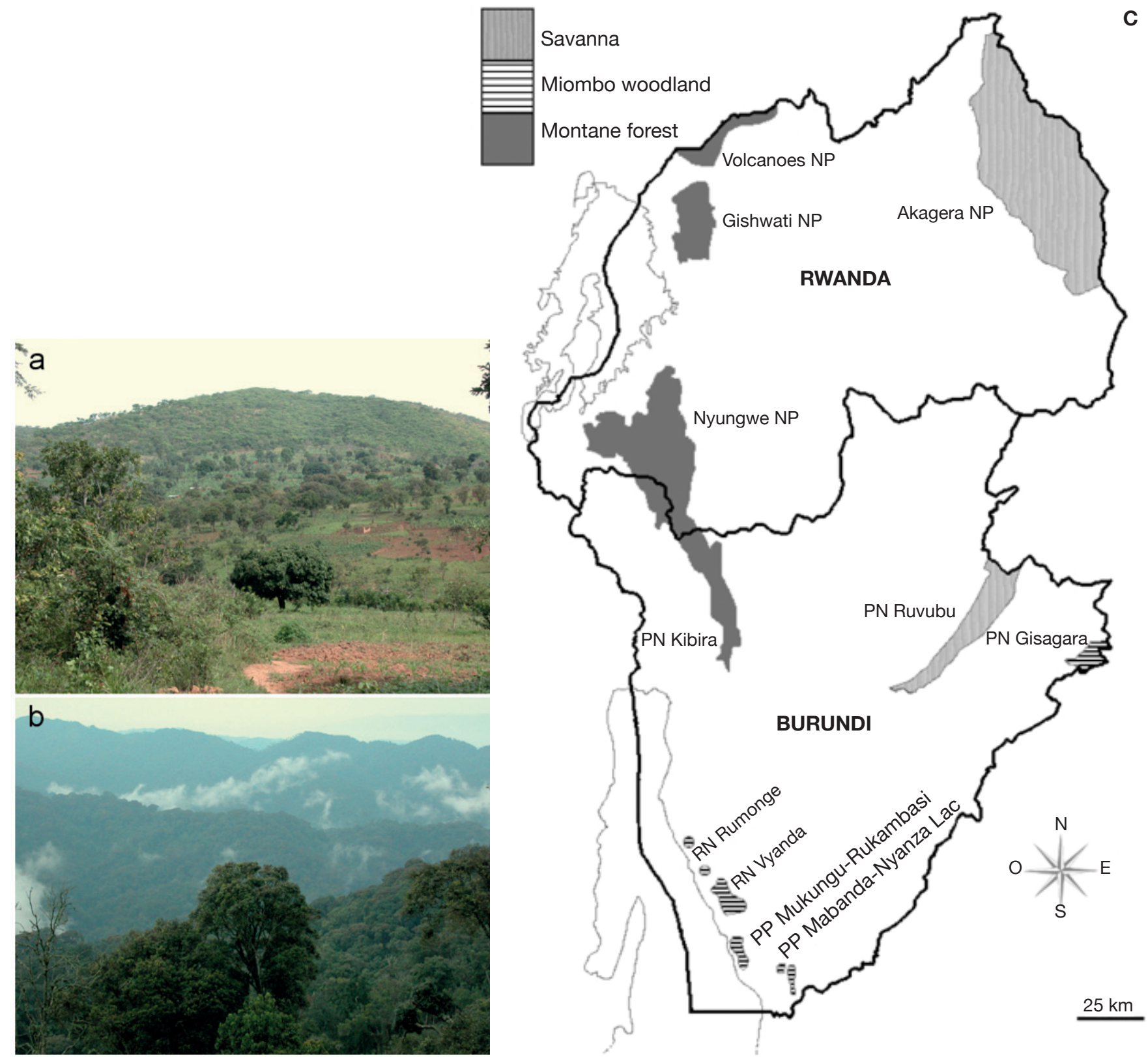

Figure 1. a. Miombo woodland on hillside in "Paysage Protégé de Gisagara" (Burundi) - forêt claire de type miombo sur une colline du "Paysage Protégé de Gisagara »(Burundi); b. Montane forest in Nyungwe National Park (Rwanda) - forêt de montagne dans le Parc National Nyungwe (Rwanda); c. Distribution of savanna, miombo woodland, and montane forest ecosystems in Burundi and Rwanda - Distribution des savanes, des forêts claires de type miombo et des forêts de montagne au Burundi et au Rwanda (Photos: Jérôme Degreef).

Identification of reference specimens was based on macroscopic and microscopic descriptions available in the literature. Scientific names and authorities follow the Index Fungorum database, the online global fungal nomenclature (www.indexfungorum.org, accessed 15/8/2015).

Reference specimens were deposited at the herbarium "BR" of the "Botanic Garden Meise" (Belgium) (Thiers, 2015), with duplicates at "Office Burundais pour la Protection de l'Environnement" in
Bujumbura for Burundian specimens or at "Rwanda Development Board" in Kigali for Rwandan specimens.

During fieldwork in Rwanda living fungal strains were collected from a number of specimens. They were obtained from spore print and plated on malt extract agar supplemented by $2 \mathrm{ppm}$ benomyl (Benlate) and $50 \mathrm{ppm}$ chloramphenicol. They are cool-stored $\left(\sim 5^{\circ} \mathrm{C}\right)$ at Kigali Farms (Rwanda) with a duplicate culture at the MUCL/BCCM mycothèque of the University of Louvain-la-Neuve (Belgium). 


\section{RESULTS}

\subsection{Checklist of edible mushrooms}

The checklist of edible species available for the region (Buyck, 1994; Buyck et al., 1995; Nzigidahera, 2007) was based on an updated list according to current taxonomy and supplemented by specimens collected during our field trips in Burundi and Rwanda (Table 1). Seventy-seven edible species were recorded. Among them, thirty-nine are newly listed as being consumed by the local population and eight (Armillaria borealis, Armillaria cepistipes, Armillaria lutea, Cystodermella elegans, Hypholoma subviride, Lentinus retinervis, Paxillus brunneotomentosus, and Pleurotus flabellatus), originating from montane forests, are recorded for the first time as edible (Boa, 2004).

\subsection{Ectomycorrhizal fungi}

Wild ectomycorrhizal fungi abound in the miombo woodlands of Burundi where they grow in a symbiotic mutualistic association with the roots of trees, mainly from the genera Brachystegia, Julbernardia, Isoberlinia (Fabaceae - Caesalpinioideae) and Uapaca (Euphorbiaceae). Savannas, pastures and farmlands have progressively replaced woodlands during the past decades in Rwanda. The host trees and their associated fungal species have consequently also disappeared from the entire territory.

The genus Amanita generally evokes highly toxic or even deadly mushrooms. The introduction to the tropics of temperate Amanita muscaria (L.) Lam. and Amanita phalloides (Vaill. ex Fr.) Link with pine and eucalypt seedlings is worth mentioning. Besides these toxic representatives, the genus also includes some excellent edible species, like Amanita loosii, largely consumed in Burundi and commonly sold at markets. Other woodland species, like Amanita mafingensis, Amanita pudica and Amanita tanzanica are occasionally eaten. We collected Amanita rubescens in the Burundian woodlands and surprisingly in the montane forests of the Kibira National Park even though its putative host tree was not identified.

Owing to local beliefs, the wild boletes are rarely consumed in tropical Africa. Even if boletes are common in the miombo woodlands, most (except Afroboletus luteolus) are not eaten by Burundian people in contrast to their Tanzanian neighbours. Also, Boletus loosii, Phlebopus colossus, Rubinoboletus balloui, and Xerocomus subspinulosus are rarely consumed in eastern Burundi. Suillus granulatus and Suillus luteus are exotic species that have been accidentally introduced to Burundi and Rwanda in the early 1900's, probably with ectomycorrhized roots of Pinus seedlings. Both species progressively found their way into the diet of the local population and are now consumed in both countries.

Although not the most appreciated mushrooms in the region, chanterelles rank first in terms of amount consumed in Burundi. The main reasons being their quite long shelf-life and the huge quantities produced in the miombo woodlands during the rainy season (Figure 2). All chanterelles are edible: the "reddish" species (Cantharellus platyphyllus var. cyanescens, Cantharellus ruber, Cantharellus splendens, Cantharellus symoensii) are most frequently sold along roadsides and local markets; the "black" Cantharellus congolensis is eaten only occasionally in Burundi, as in most tropical African countries; the "yellow-brownish" species (Cantharellus cyanoxanthus, Cantharellus defibulatus, Cantharellus densifolius, Cantharellus pseudocibarius) are often mixed in basins and baskets, without making any distinction as to their morphology. Cantharellus miomboensis, a large, firm and fleshy species also belongs in this group. It is probably one of the most abundant chanterelles of the miombo woodlands, even though it has been confused by Buyck (1994) and Nzigidahera (2007) as a variety of a rainforest species, i.e. Cantharellus rufopunctatus var. ochraceus (Buyck et al., 2013).

The family of Russulaceae (russula and milkcaps, the latter including Lactarius and the recently established closely related genus Lactifluus) includes some delicacies, i.e. Lactifluus edulis and Russula phaeocephala being the most appreciated by the Burundian population. Abundant species, like Lactarius kabansus and Russula cellulata, are harvested in the miombo woodlands and sold at the market. Other marginal species in this family (Lactifluus densifolius, Lactifluus gymnocarpoides, Lactifluus longisporus, Russula ciliata, Russula congoana, Russula hiemisilvae, Russula roseoviolacea, Russula sejuncta) are rarely harvested by villagers but are consumed locally.

Finally, the nice yellow truffle Mycoamaranthus congolensis is characterized by its scented pinkish flesh but it is also rarely eaten.

\subsection{Termitophilic fungi}

Termitophilic fungi, all from the genus Termitomyces, are among the most appreciated in the target region and unanimously seen as a delicacy by the local population. Most species recorded in Burundi and Rwanda are abundant in the montane forests' buffer zones dominated by savannas, some being also present in Burundian woodlands. That is the case for Termitomyces robustus, the most commonly eaten termitophilic species in the region and regularly sold on markets and along the roadsides. We exceptionally collected a unique specimen of Termitomyces robustus in the Hagenia-Hypericum zone (2,900 $\mathrm{m}$ a.s.1.) on 
Table 1. Edible mushrooms from Burundi and Rwanda - Champignons comestibles du Burundi et du Rwanda.

\begin{tabular}{|c|c|c|c|c|c|}
\hline & Habitat & Ecology & Country & References & $\begin{array}{l}\text { Additional } \\
\text { specimens }\end{array}$ \\
\hline $\begin{array}{l}\text { Afroboletus luteolus (Heinem.) Pegler \& } \\
\text { T.W.K. Young }\end{array}$ & $\mathrm{W}$ & $\mathrm{EM}$ & Burundi & $\begin{array}{l}\text { Buyck, 1994; } \\
\text { Nzigidahera, } 2007\end{array}$ & JD671 \\
\hline Agaricus arvensis Schaeff. & $\mathrm{Sv}$ & $\mathrm{S}$ & Rwanda & Present study & JD1334* \\
\hline Agaricus sylvicola (Vitt.) Lév. & $\mathrm{F}$ & $\mathrm{S}$ & Rwanda & Present study & JD1205 \\
\hline Amanita loosii Beeli & $\mathrm{W}$ & $\mathrm{EM}$ & Burundi & $\begin{array}{l}\text { Buyck, } 1994 \text {; } \\
\text { Nzigidahera, } 2007\end{array}$ & JD695 \\
\hline Amanita mafingensis Härk. \& Saarim. & $\mathrm{W}$ & $\mathrm{EM}$ & Burundi & Present study & JD1156 \\
\hline Amanita pudica (Beeli) Walleyn & $\mathrm{W}$ & $\mathrm{EM}$ & Burundi & $\begin{array}{l}\text { Buyck, } 1994 \text {; } \\
\text { Nzigidahera, } 2007\end{array}$ & JD699 \\
\hline Amanita rubescens Pers. & $\mathrm{F}, \mathrm{W}$ & $\mathrm{EM}$ & Burundi & $\begin{array}{l}\text { Buyck, } 1994 \text {; } \\
\text { Nzigidahera, } 2007\end{array}$ & JD1164 \\
\hline Amanita tanzanica Härk. \& Saarim. & $\mathrm{W}$ & $\mathrm{EM}$ & Burundi & Present study & JD1155 \\
\hline Armillaria borealis Marxmüller \& Korhonen & $\mathrm{F}$ & $\mathrm{S}, \mathrm{P}$ & Rwanda & Present study & $\mathrm{JD} 1312 *$ \\
\hline Armillaria cepistipes Velen. & $\mathrm{F}$ & S, P & Rwanda & Present study & JD1314* \\
\hline Armillaria heimii Pegler & $\mathrm{F}$ & $\mathrm{S}, \mathrm{P}$ & Rwanda & Present study & JD1191 \\
\hline Armillaria lutea Gillet & $\mathrm{F}$ & $\mathrm{S}, \mathrm{P}$ & Rwanda & Present study & JD1284* \\
\hline Armillaria ostoyae (Romagn.) Herink & $\mathrm{F}$ & $\mathrm{S}, \mathrm{P}$ & Rwanda & Present study & $\mathrm{JD} 1372 *$ \\
\hline Armillaria tabescens (Scop.) Emel & $\mathrm{F}$ & $\mathrm{S}, \mathrm{P}$ & Rwanda & Present study & JD1369* \\
\hline Auricularia auricula-judae (Bull.) Quél. & $\mathrm{F}$ & $\mathrm{S}$ & Rwanda & Present study & JD1258* \\
\hline Auricularia cornea Ehrenb. & $\mathrm{F}$ & $\mathrm{S}$ & $\begin{array}{l}\text { Burundi, } \\
\text { Rwanda }\end{array}$ & Present study & $\begin{array}{l}\text { JD1134, } \\
\text { JD1265* }\end{array}$ \\
\hline Auricularia delicata (Mont. ex Fr.) Henn. & $\mathrm{F}$ & $\mathrm{S}$ & $\begin{array}{l}\text { Burundi, } \\
\text { Rwanda }\end{array}$ & Present study & $\begin{array}{l}\text { JD1127, } \\
\text { JD1210* }\end{array}$ \\
\hline Boletus loosii Heinem. & $\mathrm{W}$ & $\mathrm{EM}$ & Burundi & Nzigidahera, 2007 & JD661 \\
\hline Cantharellus congolensis Beeli & $\mathrm{W}$ & $\mathrm{EM}$ & Burundi & $\begin{array}{l}\text { Buyck, } 1994 \text {; } \\
\text { Nzigidahera, } 2007\end{array}$ & \\
\hline $\begin{array}{l}\text { Cantharellus cyanoxanthus R. Heim ex } \\
\text { Heinem. }\end{array}$ & $\mathrm{W}$ & $\mathrm{EM}$ & Burundi & $\begin{array}{l}\text { Buyck, } 1994 \text {; } \\
\text { Nzigidahera, } 2007\end{array}$ & \\
\hline $\begin{array}{l}\text { Cantharellus defibulatus (Heinem.) Eyssart. \& } \\
\text { Buyck }\end{array}$ & $\mathrm{W}$ & $\mathrm{EM}$ & Burundi & $\begin{array}{l}\text { Buyck, } 1994 \text { as } \\
\text { Cantharellus cibarius } \\
\text { var. defibulatus Heinem. }\end{array}$ & \\
\hline Cantharellus densifolius Heinem. & $\mathrm{W}$ & $\mathrm{EM}$ & Burundi & Buyck, 1994 & \\
\hline Cantharellus miomboensis Buyck \& V. Hofst. & $\mathrm{W}$ & EM & Burundi & $\begin{array}{l}\text { Buyck, } 1994 \text {; } \\
\text { Nzigidahera, } 2007 \text { as } \\
\text { Cantharellus } \\
\text { rufopunctatus var. } \\
\text { ochraceus Heinem. }\end{array}$ & \\
\hline $\begin{array}{l}\text { Cantharellus platyphyllus Heinem. var. } \\
\text { cyanescens (Buyck) Eyssart. \& Buyck }\end{array}$ & $\mathrm{W}$ & $\mathrm{EM}$ & Burundi & $\begin{array}{l}\text { Buyck, } 1994 \text {; } \\
\text { Nzigidahera, } 2007 \text { as } \\
\text { Cantharellus cyanescens } \\
\text { Buyck }\end{array}$ & \\
\hline Cantharellus pseudocibarius Henn. & $\mathrm{W}$ & $\mathrm{EM}$ & Burundi & $\begin{array}{l}\text { Buyck, } 1994 \text {; } \\
\text { Nzigidahera, } 2007\end{array}$ & JD1154 \\
\hline Cantharellus ruber Heinem. & $\mathrm{W}$ & $\mathrm{EM}$ & Burundi & $\begin{array}{l}\text { Buyck, } 1994 ; \\
\text { Nzigidahera, } 2007\end{array}$ & \\
\hline
\end{tabular}


Table 1 (continued 1). Edible mushrooms from Burundi and Rwanda - Champignons comestibles du Burundi et du Rwanda.

\begin{tabular}{|c|c|c|c|c|c|}
\hline & Habitat & Ecology & Country & References & $\begin{array}{l}\text { Additional } \\
\text { specimens }\end{array}$ \\
\hline Cantharellus splendens Buyck & $\mathrm{W}$ & EM & Burundi & $\begin{array}{l}\text { Buyck, } 1994 \text {; } \\
\text { Nzigidahera, } 2007\end{array}$ & JD1152 \\
\hline Cantharellus symoensii Heinem. & $\mathrm{W}$ & EM & Burundi & $\begin{array}{l}\text { Buyck, } 1994 \text {; } \\
\text { Nzigidahera, } 2007\end{array}$ & \\
\hline Collybia aurea (Beeli) Pegler & $\mathrm{F}$ & $\mathrm{S}$ & $\begin{array}{l}\text { Burundi, } \\
\text { Rwanda }\end{array}$ & $\begin{array}{l}\text { Buyck, } 1994 \text {; } \\
\text { Nzigidahera, } 2007\end{array}$ & JD1412* \\
\hline Cotylidia aurantiaca (Pat.) A.L. Welden & $\mathrm{F}$ & $\mathrm{S}$ & $\begin{array}{l}\text { Burundi, } \\
\text { Rwanda }\end{array}$ & Present study & $\begin{array}{l}\text { JD1125, } \\
\text { JD1315* }\end{array}$ \\
\hline Cystodermella elegans (Beeli) Harmaja & $\mathrm{F}$ & S & Rwanda & Present study & JD1320 \\
\hline $\begin{array}{l}\text { Dacryopinax spathularia (Schwein.) G.W. } \\
\text { Martin }\end{array}$ & $\mathrm{F}$ & $\mathrm{S}$ & Rwanda & Present study & JD1224 \\
\hline $\begin{array}{l}\text { Hypholoma subviride (Berk. \& M.A. Curtis) } \\
\text { Dennis }\end{array}$ & $\mathrm{F}$ & $\mathrm{S}$ & $\begin{array}{l}\text { Burundi, } \\
\text { Rwanda }\end{array}$ & Present study & $\begin{array}{l}\text { JD1128, } \\
\text { JD1182* }\end{array}$ \\
\hline Lactarius kabansus Pegler \& Piearce & $\mathrm{W}$ & EM & Burundi & $\begin{array}{l}\text { Buyck, } 1994 \text {; } \\
\text { Nzigidahera, } 2007\end{array}$ & \\
\hline Lactifluus densifolius Verbeken \& Karhula & $\mathrm{W}$ & EM & Burundi & $\begin{array}{l}\text { Buyck, } 1994 \text {; } \\
\text { Nzigidahera, } 2007 \text { as } \\
\text { Lactarius inversus Gooss.- } \\
\text { Font. \& R.Heim }\end{array}$ & \\
\hline Lactifluus edulis (Verbeken \& Buyck) Buyck & $\mathrm{W}$ & EM & Burundi & $\begin{array}{l}\text { Buyck, } 1994 \text {; } \\
\text { Nzigidahera, } 2007 \text { as } \\
\text { Lactarius edulis Verbeken } \\
\text { \& Buyck }\end{array}$ & \\
\hline $\begin{array}{l}\text { Lactifluus gymnocarpoides (Verbeken) } \\
\text { Verbeken }\end{array}$ & $\mathrm{W}$ & EM & Burundi & $\begin{array}{l}\text { Nzigidahera, } 2007 \text { as } \\
\text { Lactarius gymnocarpoides } \\
\text { Verbeken }\end{array}$ & JD1150 \\
\hline Lactifluus luteopus Verbeken & $\mathrm{W}$ & EM & Burundi & Present study & JD1147 \\
\hline Lactifluus longisporus (Verbeken) Verbeken & $\mathrm{W}$ & EM & Burundi & $\begin{array}{l}\text { Nzigidahera, } 2007 \text { as } \\
\text { Lactarius longisporus } \\
\text { Verbeken }\end{array}$ & \\
\hline Lentinus cladopus Lév. & $\mathrm{F}$ & $\mathrm{S}$ & Rwanda & Present study & JD1219* \\
\hline Lentinus sajor-caju (Fr.) Fr. & $\mathrm{F}$ & $\mathrm{S}$ & Rwanda & Present study & JD1274 \\
\hline Lentinus retinervis Pegler & $\mathrm{F}$ & $\mathrm{S}$ & Rwanda & Present study & JD1269 \\
\hline Lepista sordida (Schumach.) Singer & $\mathrm{F}$ & $\mathrm{S}$ & Rwanda & Present study & JD1381* \\
\hline Macrolepiota africana (R. Heim) Heinem. & $\mathrm{F}$ & $\mathrm{S}$ & Rwanda & Present study & JD1181* \\
\hline $\begin{array}{l}\text { Macrolepiota dolichaula (Berk. \& Broome) } \\
\text { Pegler \& R.W. Rayner }\end{array}$ & $\mathrm{F}$ & $\mathrm{S}$ & Rwanda & Present study & JD1260* \\
\hline Marasmiellus inoderma (Berk.) Singer & $\mathrm{F}$ & S & Rwanda & Present study & JD1223 \\
\hline Marasmius arborescens (Henn.) Beeli & $\mathrm{F}$ & $\mathrm{S}$ & Rwanda & Present study & JD1220* \\
\hline Marasmius bekolacongoli Beeli & $\mathrm{F}$ & $\mathrm{S}$ & $\begin{array}{l}\text { Burundi, } \\
\text { Rwanda }\end{array}$ & Present study & $\begin{array}{l}\text { JD1132, } \\
\text { JD1341* }\end{array}$ \\
\hline $\begin{array}{l}\text { Mycoamaranthus congolensis (Dissing \& M. } \\
\text { Lange) Castellano \& Walleyn }\end{array}$ & $\mathrm{W}$ & EM & Burundi & $\begin{array}{l}\text { Nzigidahera, } 2007 \text { as } \\
\text { Dendrogaster congolensis } \\
\text { Dissing \& M. Lange }\end{array}$ & \\
\hline
\end{tabular}


Table 1 (continued 2). Edible mushrooms from Burundi and Rwanda - Champignons comestibles du Burundi et du Rwanda.

\begin{tabular}{|c|c|c|c|c|c|}
\hline & Habitat & Ecology & Country & References & $\begin{array}{l}\text { Additional } \\
\text { specimens }\end{array}$ \\
\hline $\begin{array}{l}\text { Paxillus brunneotomentosus Heinem. \& } \\
\text { Rammeloo }\end{array}$ & $\mathrm{F}$ & S & Rwanda & Present study & JD1232* \\
\hline Phlebopus colossus (R.Heim) Singer & $\mathrm{W}$ & $\mathrm{EM}$ & Burundi & Present study & JD1146 \\
\hline Pleurotus cystidiosus O.K. Mill. & $\mathrm{F}$ & S & $\begin{array}{l}\text { Burundi, } \\
\text { Rwanda }\end{array}$ & Buyck, 1994 & $\begin{array}{l}\text { JD1130, } \\
\text { JD1283* }\end{array}$ \\
\hline Pleurotus djamor (Rumph. ex Fr.) Boedijn & $\mathrm{F}$ & $\mathrm{S}$ & Rwanda & Present study & JD1344* \\
\hline Pleurotus flabellatus Sacc. & $\mathrm{F}$ & S & Rwanda & Present study & JD1203* \\
\hline Pleurotus tuber-regium (Fr.) Singer & $\mathrm{W}$ & $\mathrm{S}$ & Burundi & Buyck, 1994 & \\
\hline Polyporus tenuiculus (P. Beauv.) Fr. & $\mathrm{F}$ & S & Rwanda & Present study & JD1375* \\
\hline Psathyrella atroumbonata Pegler & $\mathrm{F}$ & $\mathrm{S}$ & Rwanda & Present study & JD1335* \\
\hline Psathyrella tuberculata (Path.) A.H. Sm. & $\mathrm{F}$ & S & Rwanda & Present study & JD1393 \\
\hline $\begin{array}{l}\text { Rubinoboletus balloui (Peck) Heinem. \& } \\
\text { Rammeloo }\end{array}$ & $\mathrm{W}$ & $\mathrm{EM}$ & Burundi & Nzigidahera, 2007 & JD677 \\
\hline Russula cellulata Buyck & $\mathrm{W}$ & $\mathrm{EM}$ & Burundi & $\begin{array}{l}\text { Buyck, } 1994 \text {; Nzigidahera, } \\
2007\end{array}$ & JD1167 \\
\hline Russula ciliata Buyck & $\mathrm{W}$ & $\mathrm{EM}$ & Burundi & Present study & JD1175 \\
\hline Russula congoana Pat. & $\mathrm{W}$ & EM & Burundi & Present study & JD1149 \\
\hline Russula hiemisilvae Buyck & $\mathrm{W}$ & $\mathrm{EM}$ & Burundi & Present study & JD1174 \\
\hline Russula phaeocephala Buyck & $\mathrm{W}$ & $\mathrm{EM}$ & Burundi & Buyck, 1994 & \\
\hline Russula roseoviolacea Buyck & $\mathrm{W}$ & EM & Burundi & Present study & JD1173 \\
\hline Russula sejuncta Buyck & $\mathrm{W}$ & EM & Burundi & Nzigidahera, 2007 & \\
\hline Schizophyllum commune Fr. & $\mathrm{F}$ & $\mathrm{S}$ & Burundi & Nzigidahera, 2007 & \\
\hline Suillus granulatus (L.) Roussel & $\mathrm{P}$ & EM & Rwanda & Present study & AM27 \\
\hline Suillus luteus (L.) Roussel & $\mathrm{P}$ & $\mathrm{EM}$ & Burundi & Buyck, 1994 & \\
\hline Termitomyces letestui (Pat.) R. Heim & $\mathrm{W}, \mathrm{Sv}$ & $\mathrm{T}$ & Burundi & $\begin{array}{l}\text { Buyck, } 1994 \text {; Nzigidahera, } \\
2007\end{array}$ & \\
\hline Termitomyces mammiformis R. Heim & $\mathrm{W}, \mathrm{Sv}$ & $\mathrm{T}$ & Burundi & Nzigidahera, 2007 & JD1162 \\
\hline $\begin{array}{l}\text { Termitomyces microcarpus (Berk. \& Broome) } \\
\text { R. Heim }\end{array}$ & $\mathrm{W}, \mathrm{Sv}$ & $\mathrm{T}$ & $\begin{array}{l}\text { Burundi, } \\
\text { Rwanda }\end{array}$ & $\begin{array}{l}\text { Buyck, } 1994 \text {; Nzigidahera, } \\
2007\end{array}$ & AM41 \\
\hline Termitomyces robustus (Beeli) R. Heim & $\mathrm{F}, \mathrm{Sv}$ & $\mathrm{T}$ & $\begin{array}{l}\text { Burundi, } \\
\text { Rwanda }\end{array}$ & $\begin{array}{l}\text { Buyck, } 1994 ; \text { Nzigidahera, } \\
2007\end{array}$ & $\begin{array}{l}\text { JD1180, } \\
\text { JD1396 }\end{array}$ \\
\hline Termitomyces schimperi (Pat.) R. Heim & $\mathrm{Sv}$ & $\mathrm{T}$ & $\begin{array}{l}\text { Burundi, } \\
\text { Rwanda }\end{array}$ & Nzigidahera, 2007 & AM23 \\
\hline Termitomyces striatus (Beeli) R. Heim & $\mathrm{W}, \mathrm{Sv}$ & $\mathrm{T}$ & $\begin{array}{l}\text { Burundi, } \\
\text { Rwanda }\end{array}$ & $\begin{array}{l}\text { Buyck, } 1994 \text {; Nzigidahera, } \\
2007\end{array}$ & $\begin{array}{l}\text { JD1180, } \\
\text { AM24 }\end{array}$ \\
\hline Termitomyces titanicus Pegler \& Piearce & Sv & $\mathrm{T}$ & Burundi & $\begin{array}{l}\text { Buyck, } 1994 \text {; Nzigidahera, } \\
2007\end{array}$ & \\
\hline Xerocomus subspinulosus Heinem. & $\mathrm{W}$ & EM & Burundi & Nzigidahera, 2007 & JD1165 \\
\hline
\end{tabular}

$\mathrm{W}$ : miombo woodland - forêt claire de type miombo; Sv: savanna - savane; F: montane forest - forêt de montagne; P: Pinus plantation - plantation de Pinus; E: Eucalyptus plantation - plantation d'Eucalyptus; EM: ectomycorrhizal - ectomycorhizien; S: saprotrophic - saprotrophe; P: parasitic - parasite; T: termitophilic - termitophile; JD: coll. Degreef; AM: coll. Mukandera; *: living strain isolated - souche vivante isolée. 


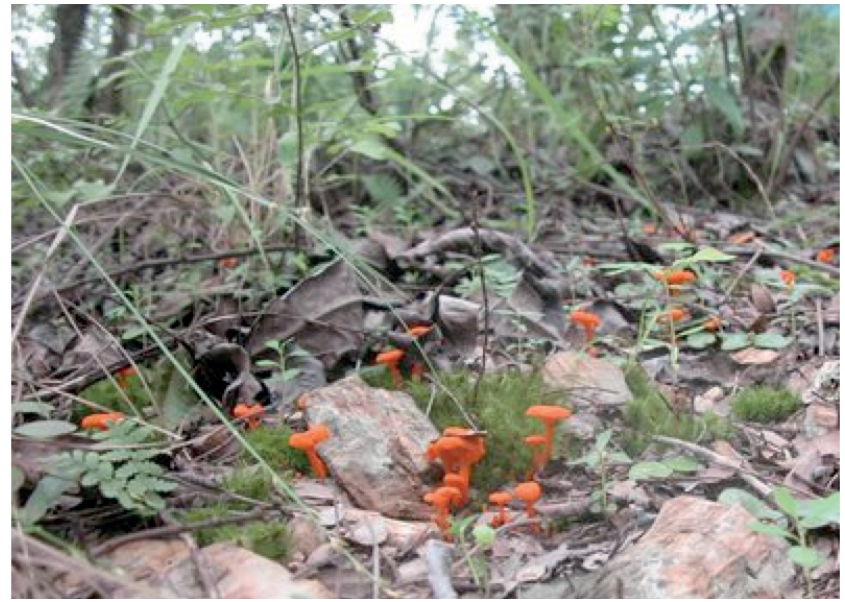

Figure 2. Ectomycorrhizal Cantharellus pseudocibarius in Brachystegia bussei, Buxus microphylla and Betula utilis miombo woodlands of the "Réserve Naturelle VyandaRumonge" (Burundi) - Cantharellus pseudocibarius, une espèce ectomycorhizienne des forêts claires de type miombo à Brachystegia bussei, Buxus microphylla et Betula utilis de la "Réserve Naturelle Vyanda-Rumonge» (Burundi). (Photo: Jérôme Degreef).

the slopes of Karisimbi volcano in Rwanda. This is the only specimen of the genus ever found in the montane forests to our knowledge (Figure 3). Another market delicacy is Termitomyces microcarpus producing large amounts of tiny fruit bodies that deteriorate very quickly (Figure 4). Other species occur less frequently (Termitomyces letestui, Termitomyces mammiformis, Termitomyces schimperi, Termitomyces striatus, and the giant Termitomyces titanicus).

\subsection{Saprotrophic fungi}

Where available, ectomycorrhizal and termitophilic edible species are generally preferred to saprotrophic mushrooms by the local population. However, with the decline of the woodlands, villagers have moved to saprotrophic species growing on dead organic materials, decomposing plant litter and decaying wood.

Ectomycorrhizal host trees and Termitomyces are generally lacking in the montane forests of Burundi and Rwanda. Only saprotrophic fungi are available for consumption. Prior to the restricted access in the recently-established National Parks, many saprotrophic species were collected and eaten by the Batwa community. Among them are representatives of genera well known for their edibility, i.e. oyster mushrooms (Pleurotus cystidiosus, Pleurotus djamor, Pleurotus flabellatus) and numerous other wood-decayers, e.g. Polyporus tenuiculus, the jelly fungi (Auricularia auricula-judae, Auricularia cornea, Auricularia delicata, Dacryopinax spathularia), the reviviscent split gill fungus (Schizophyllum commune), lentins

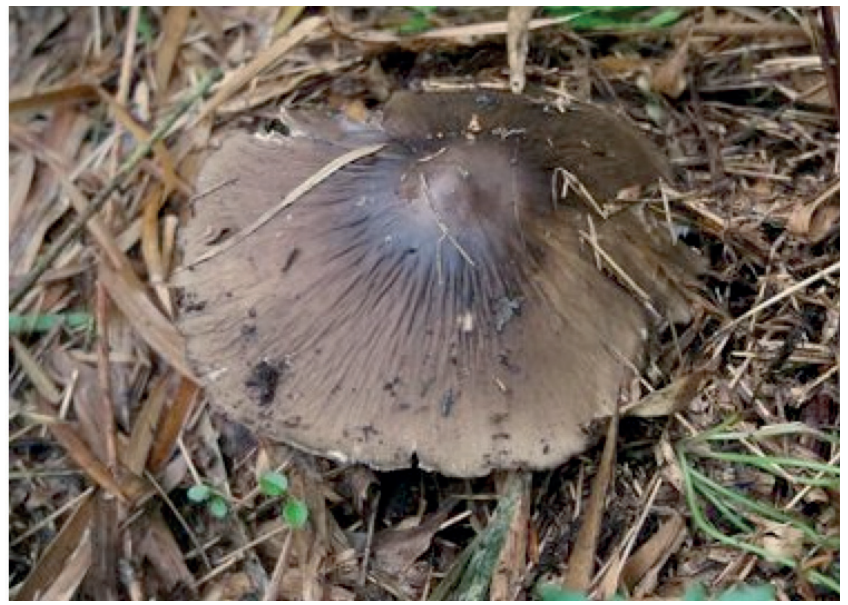

Figure 3. Termitomyces robustus first recorded on the slopes of Karisimbi volcano in the "Volcanoes National Park" (Rwanda) - Première découverte de Termitomyces robustus sur les flancs du volcan Karisimbi dans le "Parc National des Volcans » (Rwanda) (Photo: Jérôme Degreef).

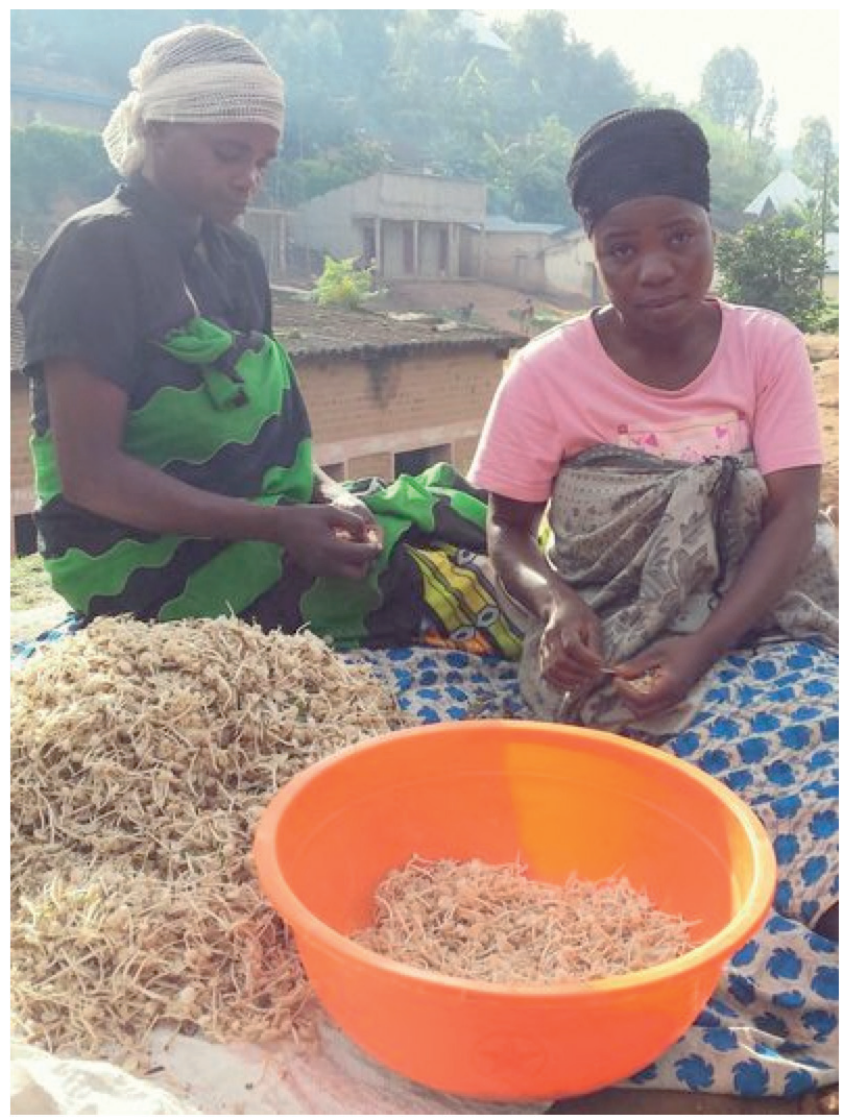

Figure 4. Termitomyces microcarpus on sale near "Gishwati National Park" (Rwanda) - Termitomyces microcarpus en vente à proximité $d u$ "Parc National Gishwati » (Rwanda). (Photo: Franck Hidvegi). 
(Lentinus cladopus, Lentinus retinervis, Lentinus sajorcaju), and the king tuber mushroom (Pleurotus tuberregium). Honey mushrooms (Armillaria cepistipes, Armillaria heimii, Armillaria lutea, Armillaria ostoyae, Armillaria tabescens) parasitize trees in montane forest and decompose the wood once the host is death. Cystodermella elegans, until now only known from DR Congo and East-African mountains, has been recorded for the first time as edible in Rwanda.

On the contrary, some species commonly eaten in neighboring countries are not consumed in Burundi and Rwanda despite being abundant in the region. This is the case for the temperate Agaricus sylvicola and Agaricus arvensis, the latter was collected in the higher altitude savannas of Rwanda. Litter-decaying parasol mushrooms (Macrolepiota africana, Macrolepiota dolichaula), Marasmius arborescens and Marasmius bekolacongoli are popular foods in the nearby Kivu province (DR Congo) but not harvested by the local population in Burundi and Rwanda. The highly palatable Lepista sordida is also worth mentioning from the slopes of the Rwandan Sabyinyo volcano. This temperate saprotrophic edible species grows on decomposing bamboo litter of Yushania alpina (K.Schum.) W.C.Lin.

Information recorded from our local guides and data collected from the villagers in the vicinity of the National Parks revealed four favorite edible species from montane forests (Figure 5):

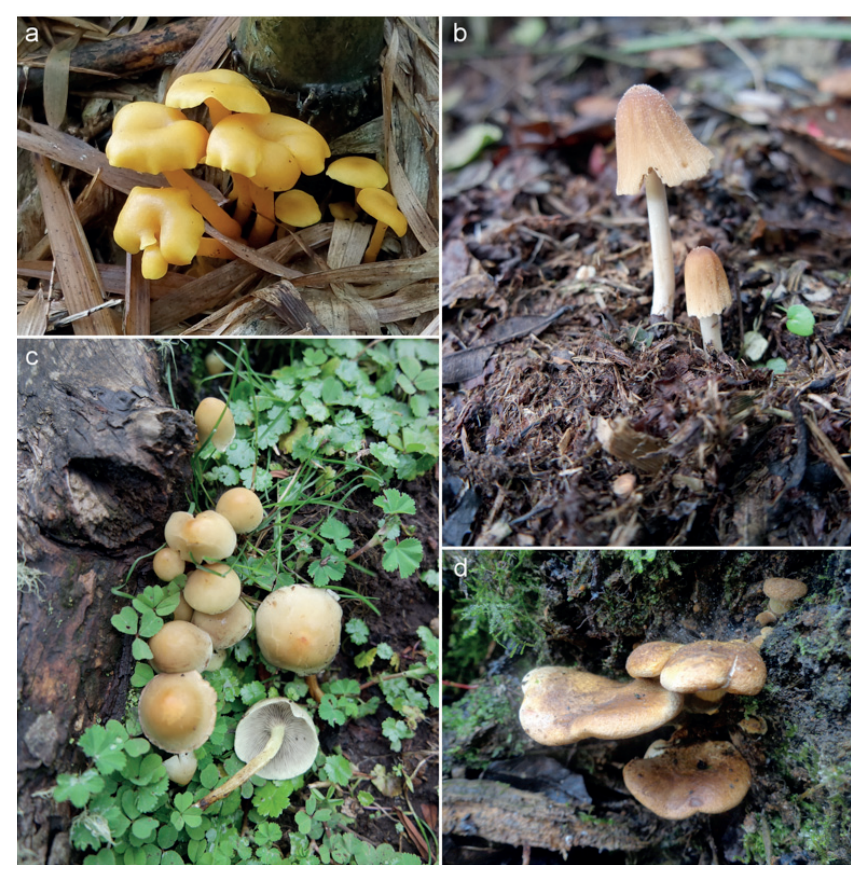

Figure 5. Favourite edible saprotrophic mushrooms Espèces saprotrophes comestibles les plus appréciées (Photos: Jérôme Degreef).

a. Collybia aurea; b. Psathyrella atroumbonata; c. Hypholoma subviride; d. Paxillus brunneotomentosus.
- Collybia aurea grows in tufts on rotten tree stumps or decomposing the Yushania alpina bamboo sticks on the slopes of the Rwandan volcanoes. This species is eaten in Burundi (Buyck, 1994), and is also consumed and sold in Rwandan villages;

- Psathyrella atroumbonata grows in small tufts on dead roots and rotten trees in the montane forests. This edible species, well-known in West-African countries, is also eaten in Rwanda;

- the bitter-tasty Hypholoma subviride grows in large clumps on stumps and rotting trunks of the montane forests. Its edibility has been recorded for the first time, despite being closely related to the poisonous sulphur tuft (Hypholoma fasciculare [Huds.] P.Kumm.) widespread in northern Europe and North America;

- Paxillus brunneotomentosus is a rare fleshy species restricted to rotten tree stumps in the montane forests. Its use as food is reported here for the first time. This is an unexpected observation since its close relatives from the Northern hemisphere, i.e. Paxillus involutus (Batsch) Fr. and allies, are known to cause severe intoxication or death when consumed on a regular basis.

\section{DISCUSSION AND CONCLUSIONS}

\subsection{Conservation of fungi in natural ecosystems}

Considering both the nutritional and economical value of wild edible mushrooms for the local population and the small remaining areas of natural ecosystems harboring these fungi in Burundi and Rwanda, the extinction risk for every taxa is worth assessing. As previously underlined by Yorou et al. (2011), most IUCN criteria for establishing a national or regional Red List are inapplicable for African fungi. Consequently, it is only the low number of localities, the restricted surface of the habitat and the present-day threats that rest upon them, that can be used for assigning threat categories.

Two hotspots of fungal diversity are clearly identifiable in the region:

- the miombo ecozone, restricted to small patches of woodlands in southern and eastern Burundi;

- the montane forest ecozone, covering larger areas straddling the borders between Burundi, Rwanda and DR Congo.

The miombo woodlands face strong human pressure, the greatest danger for the ectomycorrhizal fungi being the selective destruction and charcoaling of their host trees. As soon as the miombo is converted to farmers' fields or pasture lands, the typical ectomycorrhizal species irreparably disappeared in favor of a poorer saprotrophic mycoflora. All 36 edible ectomycorrhizal 
species listed from the miombo patches (each less than a few dozen $\mathrm{km}^{2}$ ) fit the criteria of the IUCN category "critically endangered" (CR), although some taxa remain abundant on the hillsides bordering Tanzania. Among them, Afroboletus luteolus, Cantharellus congolensis, Lactifluus edulis and Russula cellulata were already categorized "CR" in the Red List of fungi of Benin (Yorou et al., 2011).

Our findings should encourage the Burundian authorities to use the present Red List as a conservation tool, arguing in favor of stricter regulations to the access and exploitation of the so-called "paysages protégés", the last remnants of the miombo woodlands in the region. This should help to guarantee that the collecting of these fungi is done in a sustainable way that does not negatively impact the nature/structure of the miombo woodlands, nor impact mushroom yields for the future. Restoration of degraded miombo woodlands by means of ectomycorrhizal pre-inoculated local tree species, could also aid the reforestation of some Burundian and Rwandan hillsides.

The saprotrophic mycoflora of the montane forests benefits from a stronger protection in both countries with access strictly regulated by the National Parks legislations. Edible saprotrophic species are also less susceptible to extinction in the extended montane forests overlapping DR Congo where they take advantage of the limited ecosystem disturbances and low human pressure.

Finally, most of the termitophilic fungi occur in the savanna and in the buffer zones surrounding the National Parks. Because of their relatively wide distribution and the fact that destructive collecting of termite queens for consumption is not a common practice in both countries, none of the Termitomyces are assigned to a threat category.

\subsection{Wild mushrooms potential for cultivation}

Domestication of edible ectomycorrhizal fungi remains a challenge for mushroom growers worldwide. Until recently, success has been limited to a few temperate species by planting appropriate seedlings inoculated with specific fungal strains. It has been through this method that some exotic ectomycorrhizal fungi like Suillus granulatus and Suillus luteus have been accidentally introduced with pine and eucalypt seedlings to Burundi and Rwanda in the past. Isolation of most ectomycorrhizal species is problematic because they generally do not grow on artificial media thus making an inoculum difficult to produce artificially. Even in the case of effective isolation and successful inoculation, artificial production will require a specific environment with adequate soil and an optimal climate.

The mycelium of wild termitophilic fungi is grown, without exception, by fungus-growing termites. This symbiosis is the result of co-evolution between fungal species of Termitomyces specifically and termites of the subfamily Macrotermitinae. The mycelium develops on a special structure, the fungus comb, produced and maintained by the termites in fungal chambers belowground or within the depths of the termite mounds. Attempts to cultivate various termitophilic fungi, with their palatability, high protein content and great marketing potential, have been unsuccessful until present. Environmental conditions inside termite nests and the interaction with other microorganisms are suspected in the regulation of mycelial development and the triggering of their fruiting process, but the detailed process remains a mystery.

At present, saprotrophic mushrooms are the only fungi to potentially grow on an artificial medium, as such the search for new wild candidates should be looked for in this group. We attempted to isolate the related living strain on artificial medium for all saprotrophic mushrooms collected during our fieldwork. Isolation and mycelium growth succeeded in Petri dishes for 24 species (Table 1). The availability of suitable substrates is sometimes a limiting factor for successful mushroom cultivation. This is not the case for the target region, where agricultural by-products and agro-industrial wastes are abundant. Leftover cotton-seeds, sugar cane bagasse, wheat, rice, maize or bean by-products, but also composted wild savanna grasses, bamboo leaf-litter or sticks could be used for mushroom cultivation. These native species grow in the montane forests at cool temperatures and high humidity. These optimal conditions prevail for the larger part of the year, especially in the highland areas of Burundi and Rwanda. Convincing results of domestication were recently obtained with the fruiting of wild Rwandan strains of Pleurotus cystidiosus and Pleurotus flabellatus (Figure 6) by Kigali Farms, a private company operating locally. Further tests should be conducted in order to obtain additional substrates for growing some marginal, but popular, edible species having a high market potential.

\subsection{Food security and business perspectives}

Mushrooms are rich in proteins, essential amino-acids, minerals such as zinc and iron, and various vitamins. Their commercial cultivation essentially upcycles agri-waste into a unique and highly nutritious food. Mushrooms do not require good soils, as they are grown on transformed agri-waste. The recycling nature of this activity results in a minimal ecological footprint, making it very suitable for smallholder farmers. As mushrooms can be grown all year round, independent of season, their cultivation provides cash flow throughout the year and protects against weather instability. The generated income is very welcome in 


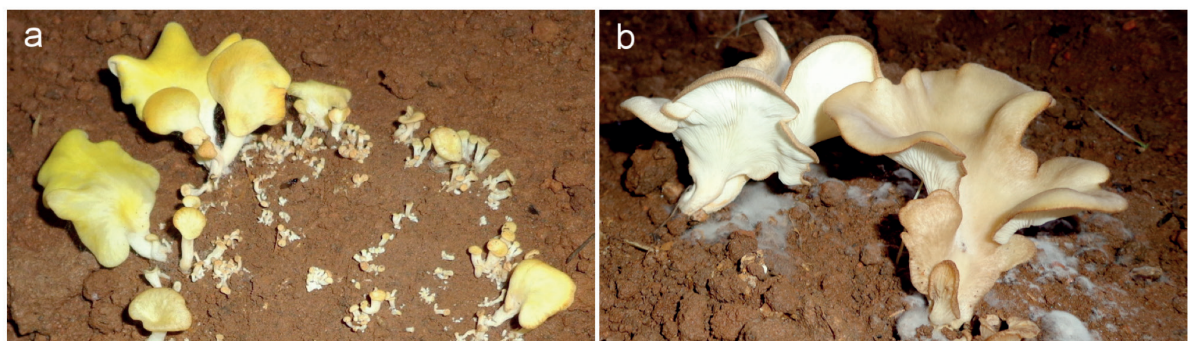

Figure 6. Fruiting from wild strains collected in Rwandan montane forests - Fructifications obtenues à partir de souches sauvages collectées dans les forêts de montagne du Rwanda (Photos: Assumpta Mukandera).

a. Pleurotus flabellatus; b. Pleurotus cystidiosus.

the run up to the harvesting periods (from March to May and September to November), especially when access to food becomes problematic, food prices go up and opportunities for employment run out. We estimate that farmers, with 0.5 ha of land (the average per inhabitant in the region), who dedicate just $1 \%$ of their land to growing mushrooms, can earn upwards of US\$ 350 per year, i.e. increasing their family budget with up to $50 \%$.

Harvesting fruit bodies in the wild remains the most appropriate strategy to benefit from edible ectomycorrhizal species. At the moment there is no organized commercial effort to collect these mushrooms for export, which would require drying facilities or a functioning cold chain. Drying mushrooms is a simple way of extending shelf life and should be encouraged, as much of the nutritional value is preserved. Dried fish is a commonly accepted source of protein in Burundi and Rwanda, but the population is not familiar with the use of dried mushrooms. Therefore, any such effort should go hand in hand with educational or promotional campaigns. Alternatively, export markets in countries where this type of transformed food is commonly used should be sought. There is significant demand for dried mushrooms in Kinshasa (DR Congo), for example, that could be supplied from Burundi or Rwanda using existing logistical channels. Organizing export of fresh edible ectomycorrhizal species from Burundian woodlands to far-flung markets such as Europe would generate a significant source of income, providing that the norms for processing and cold chain are met.

Mushrooms of Burundi and Rwanda can be a source of nutrition and income. Local consumption should be encouraged, i.e. through education about nutritional benefits and about ways to integrate them in local cooking. Alongside this, cash crop farming should be developed and its production cost-effectively shipped to market. Mushroom cultivation, which outside South Africa has not been promoted on a large commercial scale, is in fact ideally suited to smallholder farmers from the cooler parts of East Africa, provided agri- waste is available for substrate production and markets are accessible. There is a demand for mushrooms from the growing middle class in Burundi, Rwanda, Kenya, Uganda, Tanzania and DR Congo which is currently partially met through importation and wild harvested mushrooms. Building up the value chain from the local communities to these markets will enable thousands of smallholders to earn a meaningful income from their harvest or crop. Identifying local wild species suitable for cultivation and to local environmental conditions will offer consumers year-round availability of seasonal delicacies, and would diversify and broaden the income potential of mushrooms in East Africa.

\section{Acknowledgements}

Field missions in Rwanda were financially supported by FONERWA in the framework of the "Developing local mushroom strains to improve smallholder outgrower livelihoods and defend against National Park encroachment" project. The Belgian Directorate-General for Development cooperation (DGD) supported the field mission in the Kibira National Park through the CEBios programme. The authors thank the Rwanda Development Board for facilitating the access to and field work in the Rwandan National Parks. The authors are also grateful to the OBPE (formerly INECN) for authorizing the collection of specimens in the Burundian Forest Reserves and National Parks. Thanks to Cyrille Gerstmans for designing the vegetation map and to Henry Engledow for checking the text.

\section{Bibliography}

Boa E., 2004. Wild edible fungi. A global overview of their use and importance to people. Non-Wood Forest Products 17. Roma: FAO.

Buyck B., 1994. Ubwoba. Les champignons comestibles de l'Ouest du Burundi. Publication agricole, 34. Brussels: AGCD.

Buyck B. \& Nzigidahera B., 1995. Ethnomycological notes from Western Burundi. Belg. J. Bot., 128(2), 131-138.

Buyck B., Laessoe T., Meyer M. \& Hofstetter V., 2010. Chapter 12. Collecting the neglected kingdom: guidelines for the field mycologist with emphasis on the larger fungi. In: Eymann J. et al., eds. Manual on field recording techniques and protocols for All Taxa Biodiversity Inventories (ATBIs). ABC Taxa, 8, 308-330.

Buyck B., Kauff F., Cruaud C. \& Hofstetter V., 2013. Molecular evidence for novel Cantharellus 
(Cantharellales, Basidiomycota) from tropical African miombo woodland and key to all tropical African chanterelles. Fungal Divers., 58, 281-298.

Chipompha N.W.S., 1985. Some mushrooms of Malawi. Zomba, Malawi: Forest Research Institute of Malawi.

De Kesel A., 2001. A mushroom dryer for the travelling mycologist. Field Mycol., 2(4), 131-133.

De Kesel A., Codjia J.-C. \& Yorou S.N., 2002. Guide des champignons comestibles du Bénin. Meise, Belgique : Jardin Botanique National de Belgique.

De Kesel A. \& Malaisse F., 2010. Edible wild food: fungi. In: Malaisse F. How to live and survive in Zambezian open forest (Miombo ecoregion). Gembloux, Belgique : Les Presses agronomiques de Gembloux, 41-56.

Degreef J., Malaisse F., Rammeloo J. \& Baudart E., 1997. Edible mushrooms of the Zambezian woodland area: a nutritional and ecological approach. Biotechnol. Agron. Soc. Environ., 1, 221-231.

Ducousso M., Bâ A.M. \& Thoen D., 2003. Les champignons ectomycorhiziens des forêts naturelles et des plantations d'Afrique de l'Ouest: une source de champignons comestibles. Bois For. Trop., 275(1), 51-63.

Eyi Ndong H. \& Degreef J., 2010. Diversité des espèces de Cantharellus, Lentinus et Termitomyces consommées par les Pygmées du Nord du Gabon. In: Van der Burgt J., van der Maesen J. \& Onana J.-M. Systématique et conservation des plantes africaines. Kew, UK : Royal Botanic Gardens, 133-141.

Eyi Ndong H., Degreef J. \& De Kesel A., 2011. Champignons comestibles des forêts denses d'Afrique centrale. Taxonomie et identification. ABC Taxa, 10.

Guissou K.M.L., Lykke A.M., Sankara P. \& Guinko S., 2008. Declining wild mushroom recognition and usage in Burkina Faso. Econ. Bot., 62(3), 530-539.

Härkönen M., Buyck B., Saarimäki T. \& Mwasumbi L., 1993. Tanzanian mushrooms and their uses. 1. Russula. Karstenia, 33, 11-50.

Härkönen M., Saarimäki T. ＆ Mwasumbi L., 1994. Tanzanian mushrooms and their uses. 4. Some reddish edible and poisonous Amanita species. Karstenia, 34, 47-60.

Härkönen M., Saarimäki T. \& Mwasumbi L., 1995. Edible mushrooms of Tanzania. Karstenia, 35(suppl.).

Härkönen M., Niemelä T. \& Mwasumbi L., 2003. Tanzanian mushrooms. Edible, harmful and other fungi. Helsinki: Finish Museum of Natural History. Norrlinia, 10.

Härkönen M. et al., 2015. Zambian mushrooms and mycology. Helsinki: Finish Museum of Natural History. Norrlinia, 29.

Karhula P. et al., 1998. Tanzanian mushrooms and their uses. 6. Lactarius. Karstenia, 38, 49-68.

Malaisse F., 1997. Approche écologique et nutritionnelle. 2.1. Les champignons. In: Se nourrir en forêt claire africaine. Wageningen, The Netherlands: CTA;
Gembloux, Belgique: Les Presses agronomiques de Gembloux, 39-53.

Malaisse F., De Kesel A., N'Gasse G. \& Lognay G., 2008. Diversité des champignons consommés par les Boffi de la Lobaye (République centrafricaine). Geo-Eco-Trop, 32, 1-8.

Morris B., 1987. Common mushrooms of Malawi. Oslo: Fungiflora.

Morris B., 1990. An annotated check-list of the macrofungi of Malawi. Kirkia, 13, 323-364.

Mossebo D.C., Amougou A. \& Atangana R.E., 2002. Contribution à l'étude du genre Termitomyces (Basidiomycètes) au Cameroun. Écologie et systématique. Bull. Soc. Mycol. Fr., 118, 195-249.

Nzigidahera B., 2007. Ressources biologiques sauvages du Burundi. État des connaissances traditionnelles. Bujumbura : INECN.

Pegler D.N. \& Piearce G.D., 1980. The edible mushooms of Zambia. Kew Bull., 35, 475-491.

Piearce G.D., 1981. Zambian mushrooms, customs and folklore. Bull. Br. Mycol. Soc., 15, 139-142.

Rammeloo J.\& Walleyn R., 1993. The edible fungi of Africa south of the Sahara. A literature survey. Scr. Bot. Belg., 5.

Thiers B., 2015 (continuously updated). Index Herbariorum: a global directory of public herbaria and associated staff. New York, NY, USA: New York Botanical Garden's Virtual Herbarium, http://sweetgum.nybg.org/ $\mathrm{ih} /,(15 / 8 / 2015)$.

van Dijk H., Onguene N.A. \& Kuyper T.W., 2003. Knowledge and utilization of edible mushrooms by local populations of the rain forest of South Cameroon. Ambio, 32(1), 19-23.

Yorou N.S. \& De Kesel A., 2001a. Connaissances ethnomycologiques des peuples Nagot du centre du Bénin (Afrique de l'Ouest). Syst. Geogr. Plants, 71, 627637.

Yorou N.S., De Kesel A., Sinsin B. \& Codjia J.-C., 2001 b. Diversité et productivité des champignons comestibles de la forêt classée de Wari-Maro (Bénin, Afrique de l'Ouest). Syst. Geogr. Plants, 71, 613-625.

Yorou N.S. \& De Kesel A., 2011. Champignons supérieurs. In : Neuenschwander P., Sinsin B. \& Goergen G., eds. Protection de la nature en Afrique de l'Ouest : une liste rouge pour le Bénin. Ibadan, Nigeria : IITA, 47-60.

Yorou N.S. et al., 2014. Biodiversity and sustainable use of wild edible fungi in the Sudanian center of endemism. A plea for valorisation. In: BâA., McGuire K.L. \& Diédhiou A.G., eds. Ectomycorrhizal symbiosis in tropical and neotropical Forests. Boca Raton, FL, USA: Science Publishers, 241-269.

(35 ref.) 